Hence Cæsar's astronomer, Sosogenes, was so much out in his reckoning; but the people doubtless did not observe it, supposing that the new undertaking was begun with the new moon, in accordance with their superstitions.

No. 12 gave the results of observations from 1831 to 1895 , on R Comæ, from which it is evident that the period of this star, which was formerly 361 days, has become 365 days; and that, if the period continues to lengthen, the maxima will soon cease to be observable. If, however, we adopt a sine formula, equally well representing all the observations, we find that the period will shorten, bringing the maximum periods out of the twilight and rendering them observable. But if the period should continue to lengthen, the maximum will not be visible again for many years. Either of these theories accords perfectly well with recorded observations; but the former, depending as it does on the sine formula, seems now the more probable.

The next annual meeting of the Association will be held at Buffalo. Instead of beginning in the middle of the week, as has been the custom during the past few years, the sessions will begin on Monday and continue until Friday night. No excursions are to be given until after the close of the meeting. The President for the ensuing year will be E. D. Cope, of Philadelphia.

The officers of Section A for 1896 are as follows: VicePresident, W. E. Story, of Worcester, Mass., and Secretary, E. B. Frost, Hanover, N. H.

Levi L. Conant.

Worcester, Mass., September 14, 1895.

\title{
ON THE DIFFERENTIAL EQUATIONS OF CERTAIN SYSTEMS OF CONICS.*
}

BY MR. R. A. ROBERTS.

Is a few cases certain systems of curves can be represented very simply by differential equations of the first order. The latter, since they do not involve the parameter, give readily the tangents to curves of the system, at an arbitrary point of space or of the surface to which the curves are confined. Hence certain loci connected with the system and various properties of the curves can be obtained without having recourse to the integral. A remarkable example of this is to be found in the case of the geodesics on a quadric, all whose

* Read before the American Mathematical Society at its Second Summer Meeting, Springfield, Mass., August 28, 1895. 
properties are obtained by means of the differential equation of the first order, without having recourse to the transcendental final integral.

A short review of the kind of differential equations that I propose to investigate may be of interest.

Considering the complex variable $z_{1}=x+i y, z_{2}=x-i y$ in a plane, we have several remarkable systems comprised in the form

Thus

$$
\phi\left(z_{1}\right) d z_{1}+\phi\left(z_{2}\right) d z_{2}=0
$$

$$
\frac{d z_{1}}{z_{1}} \pm \frac{d z_{2}}{z_{2}}=0
$$

represents concentric circles and their radii;

$$
\frac{d z_{1}}{\sqrt{ }\left(c^{2}-z_{1}^{2}\right)} \pm \frac{d z_{2}}{\sqrt{ }\left(c^{2}-z_{2}^{2}\right)}=0
$$

represents confocal conics, the property that the tangents bisect the angle between the focal radii immediately following from this equation;

$$
\frac{d z_{1}}{\sqrt{\bar{Z}_{1}}} \pm \frac{d z_{2}}{\sqrt{\bar{Z}_{2}}}=0
$$

represents confocal Cartesian ovals, where

and

$$
Z=(z-a)(z-b)(z-c)
$$

$$
\frac{m d z_{1}}{\sqrt{Z_{1}}} \pm \frac{n d z_{2}}{\sqrt{Z_{2}}}=0
$$

represents confocal bicircular quartics, if $Z_{1}$ and $Z_{2}$ are biquadratic functions of $z_{1}, z_{2}$ respectively, whose roots are homographically connected, and $m, n$ are special functions of the roots. These results were, I believe, first noticed by Darboux.

Further, I may notice that

$$
\frac{d z_{1}}{\left(Z_{1}\right)^{\frac{2}{3}}}+\frac{d z_{2}}{\left(Z_{2}\right)^{\frac{2}{3}}}=0
$$

where $Z=(z-a)(z-b)(z-c)$ represents a system of curves of the sixth order, such that the three of the system passing through a given point cut at angles of $120^{\circ}$. I have investigated several properties of this system in an article contributed to vol. 15 of the Messenger of Mathematics. 
Other remarkable differential equations are those of systems of lines in elliptic co-ordinates; namely, if $p, q$ are the roots of

then

$$
\frac{x^{2}}{a+p}+\frac{y^{2}}{b+p}=1
$$

$\frac{d p}{\sqrt{ }\{(a+p)(b+p)(\alpha+p)\}} \pm \frac{d q}{\sqrt{ }\{(a+q)(b+q)(\alpha+q)\}}=0$

represents all the lines touching the conic $p+\alpha=0$; and further, if $p, q, r$ are the roots of

then

$$
\frac{x^{2}}{a+p}+\frac{y^{2}}{b+p}+\frac{z^{2}}{c+p}=1,
$$

$$
\begin{aligned}
& \Sigma \frac{d p}{\sqrt{\left\{(a+p)(b+p)(c+p)(\alpha+p)\left(\alpha^{\prime}+p\right)\right\}}}=0, \\
& \Sigma \frac{p d p}{\sqrt{\left\{(a+p)(b+p)(c+p)(\alpha+p)\left(\alpha^{\prime}+p\right)\right\}}}=0,
\end{aligned}
$$

represent all the lines touching the two confocals $p+\alpha=0$, $p+\alpha^{\prime}=0$.

The latter equations involve the first class of hyperelliptic integrals, and thus demonstrate the fact of equivalent algebraic relations. These equations, originally due to Liouville, were seen by Haedenkamp to hold for $n$ dimensions. The latter thus deduced the algebraic integrals of the whole class of hyperelliptic integrals.

Another system of differential equations may be found by using the parameters of the two tangents drawn from a point to a conic as co-ordinates. Thus if $\mu_{1}, \mu_{2}$ are the roots of $\mu^{2} x-2 \mu z+y=0$, a tangent of $z^{2}-x y=0$,

$$
\frac{d \mu_{1}}{\sqrt{M_{1}}} \pm \frac{d \mu_{2}}{\sqrt{M_{2}}}=0
$$

where $M=(\mu-a)(\mu-b)(\mu-c)(\mu-d)$, represents the system of conics touching the four tangents of $z^{2}-x y=0$, whose parameters are $a, b, c, d$. Darboux has made an extensive use of this kind of co-ordinates.

Now I propose to consider here a few forms of differential equations, similar to those just given, which represent systems of conics both in a plane and in space.

First of all, in a plane, I consider the three systems of conics having double contact with two fixed conics. Let the 
latter be referred to their common self-conjugate triangle, and let us consider the system of conics (touching four fixed lines)

$$
U_{p}=\frac{x^{2}}{a+p}+\frac{y^{2}}{b+p}+\frac{z^{2}}{c+p}=0
$$

then if $p, q$ are the roots of this equation, the differential equation

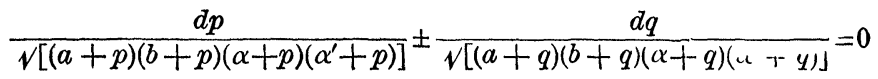

represents a system of conics having double contact with the conics obtained by putting $\alpha+p=0$ and $\alpha^{\prime}+p=0$ in (1). For, by the theory of elliptic functions, the integral of the equation can be written as a linear relation between any three of the quantities $\sqrt{ }\{(a+p)(a+q)\}, \sqrt{ }\{(b+p)(b+q)\}$, $\sqrt{ }\{(\alpha+p)(\alpha+q)\}, V\left\{\left(\alpha^{\prime}+p\right)\left(\alpha^{\prime}+q\right)\right\}$; but the first two of these quantities are proportional to $x, y$, respectively, and the last two to $\sqrt{U_{a}}$ and $\sqrt{U_{a}^{\prime}}$. Thus we get the equivalent equations

$$
\begin{array}{r}
l x+m y+n \sqrt{U_{\alpha}}=0, \\
l^{\prime} x+m^{\prime} y+n^{\prime} \sqrt{U_{a}^{\prime}}=0,
\end{array}
$$

which represent a conic having double contact with $U_{\alpha}$ and $U_{a}^{\prime}$, respectively.

Again, by using the factors $(b+p)(c+p)$ and $(a+p)(c+p)$ successively, instead of $(a+p)(b+p)$ in (2), we get the differential equations of the two other systems of conics.

As a particular case of this result, suppose we consider the ordinary plane elliptic co-ordinates, viz., those determined by

$$
\frac{x^{2}}{\mu^{2}}+\frac{y^{2}}{\mu^{2}-c^{2}}=1, \quad \frac{x^{2}}{\nu^{2}}-\frac{y^{2}}{c^{2}-v^{2}}=1
$$

then

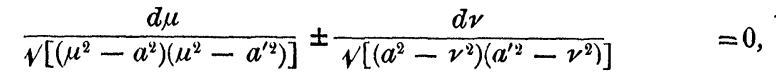

$$
\begin{aligned}
& \frac{d \mu}{\sqrt{ }\left[\left(\mu^{2}-a^{2}\right)\left(\mu^{2}-a^{\prime 2}\right)\left(\mu^{2}-c^{2}\right)\right]} \pm \frac{d \nu}{\sqrt{ }\left[\left(a^{2}-\nu^{2}\right)\left(\nu^{2}-a^{\prime 2}\right)\left(c^{2}-\nu^{2}\right)\right]}=0, \\
& \frac{\mu d \mu}{v\left[\left(\mu^{2}-a^{2}\right)\left(\mu^{2}-a^{\prime 2}\right)\left(\mu^{2}-c^{2}\right)\right]} \pm \frac{\nu d \nu}{\sqrt{ }\left[\left(a^{2}-\nu^{2}\right)\left(\nu^{2}-a^{2}\right)\left(c^{2}-\nu^{2}\right)\right]}=0,
\end{aligned}
$$

represent the three systems of conics having double contact with two confocal conics. 
If we make $a^{\prime}=\infty$, which corresponds to the two imaginary points at infinity, we get

$$
\left.\begin{array}{c}
\frac{d \mu}{\sqrt{\left(a^{2}-\mu^{2}\right)}} \pm \frac{d \nu}{\sqrt{ }\left(a^{2}-\nu^{2}\right)}=0, \\
\frac{\mu d \mu}{\sqrt{ }\left\{\left(\mu^{2}-a^{2}\right)\left(\mu^{2}-c^{2}\right)\right\}} \pm \frac{v d \nu}{\sqrt{ }\left\{\left(a^{2}-\nu^{2}\right)\left(c^{2}-\nu^{2}\right)\right\}}=0,
\end{array}\right\}
$$

as the differential equations of the two systems of circles having double contact with the conic $\mu=a$. The third equation gives in the same case the system of tangents to $\mu=\alpha$.

Proceeding now to the three dimensions, let us consider the system of quadrics (inscribed in the same developable)

$$
U_{p}=\frac{x^{2}}{a+p}+\frac{y^{2}}{b+p}+\frac{z^{2}}{c+p}+\frac{u}{d+p}=0 ;
$$

then, if $p, q, r$ are the roots of this equation,

$$
\left.\begin{array}{l}
\Sigma \frac{d p}{\sqrt{ }\left\{(\alpha+p)(b+p)(c+p)(\alpha+p)\left(\alpha^{\prime}+p\right)\left(\alpha^{\prime \prime}+p\right)\right\}}=0, \\
\Sigma \frac{p d p}{\sqrt{ }\left\{(\alpha+p)(b+p)(c+p)(\alpha+p)\left(\alpha^{\prime}+p\right)\left(\alpha^{\prime \prime}+p\right)\right\}}=0,
\end{array}\right\}
$$

represents a conic having double contact with the three quadrics $U_{a}, U_{a}{ }^{\prime}, U_{a}^{\prime \prime}$, for by the theory of the first class of hyperelliptic integrals the integrals can be expressed as linear relations between any three of the six quantities

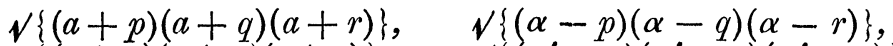

$$
\begin{aligned}
& \sqrt{ }\{(c+p)(c+q)(c+r)\}, \quad V \quad\left\{\left(\alpha^{\prime}-p\right)\left(\alpha^{\prime}-q\right)\left(\alpha^{\prime}-r\right)\right\} \text {, } \\
& \sqrt{ }\{(b+p)(b+q)(b+r)\}, \quad V\left\{\left(\alpha^{\prime \prime}-p\right)\left(\alpha^{\prime \prime}-q\right)\left(\alpha^{\prime \prime}-r\right)\right\} \text {. }
\end{aligned}
$$

Now the first three of these are proportional to $x, y$, $z$, respectively, and the three last are proportional to $\sqrt{U_{a}}, \sqrt{U_{a}^{\prime}}$, $\sqrt{U_{a}^{\prime \prime}}$. Thus we get

$$
\begin{aligned}
l x+m y+n z & =0 \\
l^{\prime} x+m^{\prime} y+n^{\prime} \sqrt{U_{a}} & =0 \\
l^{\prime \prime} x+m^{\prime \prime} y+n^{\prime \prime} \sqrt{U_{a}^{\prime}} & =0 \\
l^{\prime \prime \prime} x+m^{\prime \prime \prime} y+n^{\prime \prime \prime} \sqrt{U_{a}^{\prime \prime}} & =0
\end{aligned}
$$

and these equations obviously represent a conic having double contact with the quadrics $U_{a}, U_{a}^{\prime}, U_{a}^{\prime \prime}$.

Again, by interchanging the constants $a, b, c, d$ we obtain three other systems similar to (6). 
Thus we have demonstrated that there are four different systems of conics that can be described to have double contact with three quadrics inscribed in the same developable.

As confocal quadrics are of this kind, their equations being of the form

$$
\frac{x^{2}}{a+p}+\frac{y^{2}}{b+p}+\frac{z^{2}}{c+p}=1
$$

we obtain the differential equations of conics described to have double contact with three confocal quadrics by making $p=\infty$ in the three equations similar to (6), each of which involves $d$.

As a special case, suppose $\alpha^{\prime \prime}=\infty$, which corresponds to the imaginary circle at infinity: then the conics become circles having double contact with two confocal quadrics.

Now equations (6) in this case become simply the differential equations of lines touching the two confocals, but the three similar equations give those of the derived systems, viz.:

$$
\begin{aligned}
& \Sigma \frac{d p}{\sqrt{\left\{(a+p)(b+p)(\alpha+p)\left(\alpha^{\prime}+p\right)\right\}}}=0, \\
& \Sigma \frac{p d p}{\sqrt{\left\{(a+p)(b+p)(\alpha+p)\left(\alpha^{\prime}+p\right)\right\}}}=0,
\end{aligned}
$$

and the two other similar equations obtained by interchanging $a, b, c$. Thus there are three systems of circles which can be described to have double contact with two confocal quadrics. Furthermore, either of these quadrics can degenerate to a focal conic. Thus, for instance, since $p+a=0$, $p+b=0$ give the two focal conics, we have

$$
\begin{aligned}
& \Sigma \frac{ \pm d p}{(a+p)(b+p)}=0, \quad \Sigma \frac{ \pm p d p}{(a+p)(b+p)}=0 ; \\
& \Sigma \frac{d p}{(a+p) \sqrt{ }\{(b+p)(c+p)\}}=0, \quad \Sigma \frac{p d p}{(a+p) \sqrt{\{(b+p)(c+p)\}}}=0 \text {; } \\
& \Sigma_{\frac{d p}{(b+p) \sqrt{ }\{(a+p)(c+p)\}}}=0, \quad \Sigma_{\frac{p d p}{(b+p) \sqrt{ }\{(a+p)(c+p)\}}}=0 \text {; }
\end{aligned}
$$

as the differential equations of circles described to intersect twice each of the two focal conics.

In each of the preceding equations we have $d p: d q: d r$ $=A: \pm B: \pm C$, say; that is, there are, in consequence of the double signs, four tangents to the curves of the system that pass through a given point. Thus there are four curves of the system that pass through a point, and from the differ- 
ential equations we can find properties of these curves, and loci connected with their intersection. Thus in elliptic coordinates, the normals to the confocal quadrics being at right angles to each other, it follows that the tangents to the four conics of the aforementioned systems that pass through a point are equally inclined to the normal of one of the quadrics; that is, if $a, \beta, \gamma$ are the angles which one of the tangents makes with the normals of the confocal quadrics, we shall have $\cos \alpha, \cos \beta, \cos \gamma$ respectively proportional to $\lambda, \pm \mu, \pm \nu$, say, for the tangents of the four conics of the system. Hence, if two of the conics should cut at right angles, we should have $\lambda^{2}+\mu^{2}=\nu^{2}$, from which we can derive the equation of the locus in elliptic co-ordinates.

I now proceed to consider the problem: to find the differential equations of the system of conics touching six fixed planes in space.

Now there is a definite unique twisted curve of the third degree which may be described to osculate the six fixed planes. Hence, since an osculating plane of the twisted cubic can be written in the form

$$
X_{\mu}=\mu^{3} x+\mu^{2} y+\mu z+u=0,
$$

it follows that the six planes can be obtained by substituting six quantities $a_{1}, a_{2}, a_{3}, a_{4}, a_{5}, a_{6}$ in this equation in place of $\mu$. Now let us consider the differential equations

$$
\Sigma \frac{d \mu}{\sqrt{ } M}=0, \quad \Sigma \frac{\mu d \mu}{\sqrt{ } M}=0,
$$

where the summation refers to the parameters $\mu_{1}, \mu_{2}, \mu_{3}$ of the three osculating planes which can be drawn through a point, and

$$
M=\left(\mu-a_{1}\right)\left(\mu-a_{2}\right)\left(\mu-a_{3}\right)\left(\mu-a_{4}\right)\left(\mu-a_{b}\right)\left(\mu-a_{6}\right) .
$$

Then, by the theory of the first class of hyperelliptic integrals, we have as the integrals a series of linear relations connecting the six quantities

$$
\begin{gathered}
V\left\{\left(a_{1}-\mu_{1}\right)\left(a_{1}-\mu_{2}\right)\left(a_{1}-\mu_{3}\right)\right\}, \quad V\left\{\left(a_{2}-\mu_{1}\right)\left(a_{2}-\mu_{2}\right)\left(a_{2}-\mu_{3}\right)\right\}, \\
\cdots, V\left\{\left(a_{6}-\mu_{1}\right)\left(a_{6}-\mu_{2}\right)\left(a_{6}-\mu_{8}\right)\right\} .
\end{gathered}
$$

But, from the equation of the osculating plane, the expression $\left(a_{1}-\mu_{1}\right)\left(a_{1}-\mu_{2}\right)\left(a_{1}-\mu_{8}\right)$ is proportional to the osculating plane corresponding to the parameter $a_{1}$, and similarly for the other expressions. Thus we have linear relations connecting every three of the radicals $\sqrt{X_{a_{1}}}, \sqrt{X_{a_{2}}}, \ldots, \sqrt{X_{a_{6}}}$; 
or we may take each of these proportional to $p_{1}+q_{1} t$, $p_{3}+q_{2} t, \ldots, p_{0}+q_{6} t$, where $t$ is an arbitrary parameter. Hence we get

$\lambda X_{a_{1}}=\left(p_{1}+q_{1} t\right)^{2}, \quad \lambda X_{a_{2}}=\left(p_{2}+q_{2} t\right)^{2}, \ldots, \lambda X_{a_{6}}=\left(p_{6}+q_{\mathrm{g}} t\right)^{2}$.

Now these equations obviously represent a curve of the second degree, viz., a conic section, touching the six planes $X_{a_{1}}$, $X_{a_{2}}, \ldots, X_{a_{8}}$. Consequently the differential equation (r) represents the system of conics described to touch the six planes. Since there are four values of the ratios $d \mu_{1}: d \mu_{2}: d \mu_{3}$, it follows that there are four conics of the system passing through an arbitrary point of space.

In a paper contributed to The Proceedings of the London Mathematical Society, vol. 18, p. 202, I showed that if certain conditions were satisfied a doubly infinite number of polygons could be circumscribed about two confocal quadrics so as to have their vertices on another confocal quadric. This result was obtained by means of the theory of the first class of hyperelliptic integrals. Similar reasoning could be employed in the case of the conics that I have been considering. Thus, under certain conditions, we could have an infinite number of curvilinear polygons formed by conics having double contact with two confocal quadrics and intersecting on another confocal quadric. There would also be infinite systems of curvilinear polygons in the other cases that I have investigated.

An interesting question arises in connection with the preceding results, viz., as to the reality of the curves of the different systems. Thus, for instance, in the case of two confocal ellipses represented in elliptic co-ordinates by $\mu=a$, $\mu=a^{\prime}$, the conics belonging to the system

$$
\frac{d \mu}{\sqrt{ }\left\{\left(\mu^{2}-a^{2}\right)\left(\mu^{2}-a^{\prime 2}\right)\right\}} \pm \frac{d \nu}{\sqrt{ }\left\{\left(a^{2}-\nu^{2}\right)\left(a^{\prime 2}-\nu^{2}\right)\right\}}=0
$$

are not real, because $\mu$ must obviously lie between $a$ and $a^{\prime}$, and thus $\left(\mu^{2}-a^{2}\right)\left(\mu^{2}-a^{\prime 2}\right)$ is negative, while $\left(a^{2}-\nu^{2}\right)\left(a^{\prime 2}-\nu^{2}\right)$ is positive.

But the two other systems contain real conics, and in other cases there seem to be only two systems with real conics.

A case, however, not already noticed, in which the three systems are real, deserves to be mentioned. One of the fixed conics is, however, imaginary, namely, the imaginary circle at infinity on a sphere. Consider the confocal cones

$$
\begin{aligned}
& \frac{x^{2}}{\mu^{2}}+\frac{y^{2}}{\mu^{2}-b^{2}}=\frac{z^{2}}{c^{2}-\mu^{2}}, \\
& \frac{x^{2}}{\nu^{2}}-\frac{y^{2}}{b^{2}-\nu^{2}}=\frac{z^{2}}{c^{2}-\nu^{2}},
\end{aligned}
$$


intersecting the sphere $x^{2}+y^{2}+z^{2}=1$ in confocal spheroconics; then the differential equations

$$
\begin{aligned}
& \frac{d \mu}{\sqrt{ }\left\{\left(\mu^{2}-b^{2}\right)\left(\mu^{2}-a^{2}\right)\right\}} \pm \frac{d \nu}{\sqrt{ }\left\{\left(b^{2}-\nu^{2}\right)\left(a^{2}-\nu^{2}\right)\right\}}=0, \\
& \frac{d \mu}{\sqrt{ }\left\{\left(c^{2}-\mu^{2}\right)\left(a^{2}-\mu^{2}\right)\right\}} \pm \frac{d \nu}{\sqrt{ }\left\{\left(c^{2}-\nu^{2}\right)\left(a^{2}-\nu^{2}\right)\right\}}=0 \text {, } \\
& \frac{\mu d \mu}{\sqrt{ }\left\{\left(\mu^{2}-b^{2}\right)\left(c^{2}-\mu^{2}\right)\left(\mu^{2}-a^{2}\right)\right\}} \pm \frac{\nu d \nu}{\sqrt{ }\left\{\left(b^{2}-v^{2}\right)\left(c^{2}-\nu^{2}\right)\left(a^{2}-\nu^{2}\right)\right\}}=0 \text {, }
\end{aligned}
$$

represent the three systems of circles having double contact with the sphero-conic $\mu=\alpha$, and these it is easy to see from geometrical considerations are real. Circles of course may be considered as conics having double contact with $\mu=\infty$, or $x^{2}+y^{2}+z^{2}=0$. By projection then on a plane it would appear that we could have three real systems of conics having double contact with one real conic and one imaginary conic.

It would be interesting also to consider the reality of the conics having double contact with three quadrics. For instance, it does not seem possible to have any real conics having real double contact with three confocal ellipsoids. But I must defer the consideration of this question to another opportunity.

\section{ASYMPTOTIC LINES ON A CIRCULAR RING.*}

BY PROFESSOR H. MASCHKE.

THE equation of the asymptotic lines of a tore as well as the expression of the length of the arc of these curves leads to elliptic functions. The formulas appear in a remarkably simple form. $\dagger$

Assuming the radius $C A$ of the rotating circle $=1$, and denoting the distance $C O$ of its centre from the axis of rotation by $\lambda$, the tore is represented by these equations:

$$
x=v \cos u, \quad y=v \sin u, \quad z=\sqrt{1-(v-\lambda)^{2}},
$$

* Read before the American Mathematicar Socierty at its Secon d Summer Meeting, Springfield, Mass., August 28, 1895.

+ I have not found any literature with regard to this subject excepting a short notice in the Fortschritte der Mathematik, 1889, page 765, where reference is made to T. Motoda, "Asymptotic lines on a circular ring," Tokio Math. Soc., vol. 4, 217-219, a paper which has been inaccessible to me. 